\title{
Inmigración, diversidad cultural, reconocimiento político
}

\section{Javier de Lucas}

Universitat de València. Director de la Casa de España en París jdelucas@colesp.org

\section{Resumen}

El artículo sostiene la tesis de que, aunque la culturalización del debate migratorio tiene aspectos positivos, la prioridad se halla en la política, puesto que, si no hay participación política de los inmigrantes en la sociedad de acogida, no hay sentimiento de pertenencia, ni conciencia de identidad, y difícilmente cabe hablar de integración social de los inmigrantes.

Palabras clave: filosofía del derecho, filosofía política, migraciones, cultura.

\section{Abstract. Immigration, cultural diversity and political reconnaissance}

This article makes the claim that although a culturalist orientation in the discussion of migration has positive aspects, the real priority is in the political level, since without political participation of inmigrants in their new society, there is no feeling of belonging or identity, and therefore, it is difficult to talk about inmigrants' social integration.

Key words: philosophy of law, politics philosophy, migrations, culture.

\section{Sumario}

1. ¿Por qué es relevante la diversidad cultural que aporta la inmigración? La inmigración como cuestión política

2. La cuestión de los derechos políticos y el reconocimiento de la pluralidad constitutiva
3. Sobre la ampliación de la noción de ciudadanía

4. La participación política de los inmigrantes, clave del proceso de integración

Referencias bibliográficas 
Es casi inabarcable la literatura científica a propósito de inmigración y diversidad cultural, aunque lo cierto es que estamos cada vez más necesitados de análisis concretos, que sitúen una y otra en contextos precisos para poder detectar los problemas, las demandas y las posibles soluciones. Así sucede en particular en España, que ha pasado de forma vertiginosa a convertirse en el segundo destino de movimientos migratorios — el primero en la UE — aunque el estallido de la crisis económica pueda introducir no pocos cambios en los desplazamientos de población y, sobre todo, en las políticas de gestión de los mismos.

En cualquier caso, parece obvio que, aunque la presencia de migracion estable no es el único factor de la creciente diversidad cultural, ambos fenómenos coinciden en hacer imprescindible el examen de aquelllo que desde Hegel conocemos como dialéctica del reconocimiento, sobre todo en su dimensión jurídico-política. Algo en lo que los trabajos de Charles Taylor y Axel Honneth, entre otros, nos ayudan a avanzar. Porque creo que lo que nos interesa, o, mejor, lo que nos urge a propósito de diversidad cultural e inmigración es, sobre todo, la clave política, más que la antropológico-identitaria. Por eso, aun a riesgo de rieterativo, propongo examinar en primer lugar esta tesis, la de la condición radicalmente política del fenómeno migratorio (y la relevancia asimismo política de la diversidad cultural) al que asistimos en el contexto de transformaciones impuestas por esta etapa (y este modelo) del proceso de la globalización.

\section{1. ¿Por qué es relevante la diversidad cultural que aporta la inmigración? La inmigración como cuestión política}

Una de las tesis menos debatidas todavía acerca de la inmigración es la del profundo significado político de los modernos flujos migratorios, aunque, en realidad, es la inmigración ( $y$, obviamente, la emigración) en sí la que tiene esa dimensión radicalmente política, como ya supiera señalar Abdelamek Sayad, siguiendo a Balibar y Bourdieu, pero también subrayada con otro énfasis por Sassen o Mezzadra. Los modernos movimientos migratorios muestran a las claras la incapacidad de las viejas categorías jurídicas y políticas para abarcarlos, para gestionarlos adecuadamente y de ahí, en sentido negativo, la resistencia de la inmigración a nuestra mirada jurídica y política. Eso se debe, por decirlo de forma sumaria, al menos a tres características que son contrarias a la lógica jurídica y política de los ordenamientos jurídicos y de los estados nacionales, de las democracias construidas sobre ese molde, aunque éstos vayan siendo sustituidos o completados, al menos en el caso europeo, por una lógica, por así decirlo, regional. La primera, su globalidad. La segunda, su integralidad. La tercera, su dinamicidad, su plasticidad.

En efecto, las modernas migraciones tienen todos los rasgos del proceso de globalización y, por ello, son difícilmente abarcables desde una perspectiva estatal nacional que sigue siendo hoy la dominante en el mundo jurídico. Además, los fenómenos migratorios siguen siendo fenómenos sociales totales, 
que no pueden reducirse a una sola dimensión (la económico-laboral, la de la seguridad pública, la cultural-identitaria). Finalmente, se trata de movimientos complejos, plurales, enormemente cambiantes y que se adaptan a gran velocidad a los contextos. Basta con examinar el creciente papel de las redes que el derecho interpreta inmediatamente como mafias.

Por eso, la respuesta jurídico-política que ofrecemos ante los movimientos migratorios es, en cierto modo, la historia de un desencuentro. Los cauces a través de los cuales nuestros instrumentos jurídicos tratan de regular (en realidad, dominar) los movimientos migratorios son tan estrechos que inevitablemente se ven desbordados, y entonces surge la "natural» respuesta jurídica monista, simplificadora, la de la represión, la prohibición, la asunción de que el output básico del derecho es vigilar y castigar y hacerlo eficazmente. Es así porque nuestra respuesta jurídica arranca de una mirada sobre la inmigración que, en su propósito de encasillar a los movimientos migratorios en las categorías que le son familiares y sobre todo útiles (el trabajador en el mercado formal de trabajo), en su parti pris, deforma la realidad plural de la inmigración. Crea un concepto normativo y estrecho, simplificador, de los inmigrantes, que no sirve para tratar a la diversidad de inmigrantes reales y, sobre todo, contribuye poderosamente a su estigmatización social, al crear el fobotipo del inmigrante ilegal - hasta el extremo de la aplicación a esos inmigrantes de una vieja creación dogmático-penal de abolengo nacionalsocialista, el derecho penal del enemigo- . Por eso se propone la lucha contra la inmigración ilegal (contra la delincuencia y los peligros que de ella derivan) como tarea prioritaria del derecho ante la inmigración, de una forma que no puede dejar de calificarse como incompleta, parcial y restrictiva. Ante todo, porque la inmigración ilegal no es un factor meramente exógeno, sino que existe en función de un mercado en el que se cotiza esa mercancía, es decir, que actuar contra esa forma de criminalidad que es el tráfico y la explotación de seres humanos obliga a poner el acento no sólo (ni, diría, prioritariamente) en el control de fronteras, sino intra muros, en la tarea de inspección fiscal y laboral sobre quienes crean ese mercado, los que compran esa mercancía.

Pero, sobre todo, lo que me interesa aquí es recordar que los nuevos movimientos migratorios constituyen uno de los factores que, al tiempo que desafían nuestro discurso sobre el vínculo social y político, ofrecen oportunidades para transformarlo precisamente en el sentido del pluralismo. Son un reto para nuestras respuestas sobre quién y por qué tiene derecho a pertenecer al demos, y con ello, a nuestras categorías de ciudadanía y soberanía. Son un desafío sobre los límites de la inclusión en una democracia pluralista. Brindan argumentos para desarrollar ésta que es una de las tareas básicas de la política en el siglo en el que tan brutalmente acabamos de entrar. Y en su dimensión transnacional y no sólo global, proporcionan también medios para la construcción de lo que algunos han dado en llamar democracia postnacional y de la que tanto y tan estérilmente venimos debatiendo.

Desde el punto de vista de la gestión de la presencia de los inmigrantes, la tesis a la que quiero apuntar es que hay que reconocer que la lógica del dere- 
cho acorde con el modelo liberal, incluso allí donde su clave es la garantista (la lógica de la lucha por los derechos), es imprescindible, pero insuficiente en la gestión de la inmigración. Lo es por dos tipos de razones que tienen que ver con el concepto de pluralismo. En primer lugar, porque los inmigrantes, qua extranjeros, son el arquetipo de los excluidos (al menos los silenciados) del pacto original que define quién es sujeto del vínculo social y, por tanto, los deja fuera del pacto político. Además, porque subyace una dificultad del principio que podríamos llamar la igualdad compleja (el principio de gestión de la diferencia), que va más allá de la discriminación, pues alcanza la integración política, lo que hoy denominamos empowerment.

En realidad, el derecho no es el elemento decisivo en una política razonable de gestión de ese factor de pluralidad social que son los movimientos migratorios, en la que el elemento dirimente es una voluntad política de comprensión y orientación de la existencia de los flujos migratorios y de su presencia estable entre nosotros, pero sí es imprescindible. Por esa razón, creo que debemos pasar de la formulación restrictiva propia de un derecho defensivo frente a la inmigración (un derecho funcional a una política instrumental, economicista o a lo sumo «humanitaria»), a una política más amplia y de largo alcance, que, inevitablemente, supondrá profundas modificaciones en la mirada jurídica y política sobre los inmigrantes. El derecho, el mejor derecho, como explicara magistralmente Ihering y nos recuerda Ferrajoli, puede ambicionar ser un buen sistema de garantías de los derechos, de la igualdad en las libertades. Nada menos. Pero poco más. Quiero decir que tienen razón los que nos recuerdan que la inmigración no es sólo, ni siquiera sobre todo, una cuestión legal. Por eso es verdad que, si queremos tratar en serio la inmigración, debemos ir más allá de leyes o actuaciones judiciales.

Se repite una y otra vez — no sin razón- que el objetivo de una buena política de inmigración es la integración social y política de todos los sujetos implicados en ese proceso de interacción social, los inmigrantes y la población autóctona. Para ello es preciso, como se nos recuerda también una y otra vez, un gran acuerdo político (es lo que se llama pacto de Estado), que debe empezar por el consenso acerca de cómo regular la inmigración. Pero sobre todo es necesario, y esto no se dice casi nunca, asegurar la primera condición sin la que no puede haber integración. El reconocimiento de los derechos es esa condición. De nada valdrán los más imaginativos programas de interculturalidad, de inmersión en nuestros usos y costumbres (y en los suyos), las proclamas de tolerancia y solidaridad, si no empezamos por la igualdad en las condiciones de trabajo, en el salario, en el derecho a la salud, en el acceso a la educación. En la libertad de expresarse, asociarse y manifestarse. En el acceso a la justicia.

Siendo todo ello verdad, no podemos quedarnos ahí. Hemos hablado de condiciones previas, pero, en definitiva, si queremos profundizar en el significado radicalmente político del fenómeno de las migraciones, tenemos pendiente el reconocimiento como sujeto del espacio público de los inmigrantes, lo que exige a su vez revisar el derecho de participación política y el acceso a la ciudadanía. Y revisar también las condiciones que dificultan que ese acceso se 
produzca en términos de igualdad, sea cual fuere el punto de partida. Eso es lo que podemos denominar integración política.

\section{La cuestión de los derechos políticos y el reconocimiento de la pluralidad constitutiva}

El principio del pluralismo político, básico en democracia, exige revisar las condiciones del reconocimiento del acceso a los derechos políticos y a la ciudadanía por parte de los inmigrantes, afrontar un cambio cualitativo en la dimensión más decisiva, la más relevante, del proceso de transformación social que implica la presencia de los inmigrantes de forma estable. Un cambio que supone un paso adelante respecto al tradicional objetivo de las políticas de inmigración, centradas en los procedimientos y en los modelos de reclutamiento, circulación y primera acogida de los flujos migratorios. Como se ha dicho, ha llegado la hora de las políticas de presencia y pertenencia, que suelen remitir a un concepto tan común como las más de las veces impreciso, el de integración social u otros términos afines, como el de acomodación (Halper, Zapata). Por mi parte, entiendo que es más claro recurrir a la noción de integración política, que, en gran medida, es una manifestación de ese nuevo pluralismo que necesitamos. Una dimensión política de la integración que no entiendo como un corolario del proceso de integración social impuesto por la presencia de los flujos migratorios, sino como una exigencia básica, coherentemente con la tesis de que la inmigración es sobre todo una cuestión política, incluso el escenario privilegiado del debate político en la actualidad. Trataré de explicarme.

Como apunté más arriba, cuando hablamos de integración en el contexto de la gestión de los flujos migratorios (y lo mismo sucede incluso si empleamos nociones como incorporación, inserción o acomodación), hoy ya suele admitirse que no es sólo ni siquiera prioritariamente una cuestión de mero equilibrio del mercado de trabajo (afán que, sin duda, es acertado, pues debemos evitar ese reduccionismo presente en la base de la concepción dominante de la política de inmigración), o, al menos, que un asunto es la gestión del movimiento y, en particular, de la recepción de los flujos migratorios que llegan a nuestro país y otro diferente la regulación de la inserción social de quienes están presentes e incluso forman parte de la vida cotidiana en nuestras ciudades. Y en ese "avance» de nuestra conciencia sobre la dimensión de la integración, se da por descontada la prioridad de la dimensión sociocultural del proceso de integración. Con ello se privilegia la perspectiva de que se trata sobre todo de problemas de gestión del mantenimiento de la cohesión social en contextos de incremento relevante de la diferencia sociocultural. De esa forma, se propicia lo que entiendo como un riesgo, y es que el debate se culturaliza, si se me permite la expresión: como se sostiene que la clave de la cohesión social, de la convivencia, es la armonización de las diferencias, de la creciente pluralidad (es decir, sobre todo de la que viene de fuera a través de la inmigración), la discusión sobre integración se centra casi exclusivamente en cómo conjugar 
las diferencias culturales, es decir, en los límites del reconocimiento y la incorporación de la legítima diferencia cultural en las instituciones, las prácticas y las normas por las que se conducen las relaciones sociales. No desconozco que esa orientación nace de un punto de inflexión en cuanto a políticas de inmigración que tiene aspectos positivos:

1. Ante todo, el reconocimiento de que la integración es un proceso que exige una perspectiva global, no sectorial, y que no será el resultado automático de una aplicación normativa: el Derecho no puede asegurar procesos sociales, tampoco el de integración.

2. Además, parece ya abandonado el viejo prejuicio unidireccional e instrumental que entendía la integración como un resultado «objetivo» y como un objetivo a alcanzar por parte de los inmigrantes, sobre los que cargaba el deber de integrarse a/en la sociedad receptora y, además, de hacerlo de forma invisible, silenciosa, sin alterarla, sin producir cambios en ella. Hoy se entiende que la integración (salvo que se trate de asimilación impuesta) implica reciprocidad, negociación entre dos partes. Como ha señalado por ejemplo Maalouf y reitera Fernández Buey, eso implica para los inmigrantes el mensaje de que cuanto más se impregnen de la cultura del país de acogida, más podrán impregnarse de la propia, y para la sociedad de acogida, frente a los mensajes de asimilación impuesta como condición sine qua non de integración, la tesis de que las posibilidades de apertura de los inmigrantes a la cultura del país de recepción están en función de la percepción que a su vez tenga el inmigrado de que se respeta su cultura de origen.

3. Finalmente, hoy hemos pasado de la discusión académica a la política, en el sentido mejor de la expresión: lo que se trata ahora es de concretar las exigencias de la dimensión social del proceso de integración, o sea, debatir sobre propuestas concretas en torno a los indicadores sociales de la gestión de la convivencia plural en la vida cotidiana: de la vivienda al trabajo, pasando por la educación.

Pero creo que es otra la prioridad. Como ha señalado Fernández Buey (Inmigración: propuestas constructivas), es el momento de entender que, en lugar del dominante «rigorismo administrativo y centralizado de las falsamente llamadas "leyes de extranjería", lo que debería haber son politicas de inmigración coordinadas en la Unión Europea y políticas de inserción descentralizadas, con atención preferente a lo local, a las ciudades y a sus barrios [...] parece sensato aspirar a una política de inmigración coordinada en la Unión Europea que rompa con la práctica de las regulaciones ad hoc y deje de tratar el asunto como un problema casi exclusivamente de policía, fronteras y seguridad, para tratarlo como un asunto de derechos y ciudadanía».

En realidad, de acuerdo con el mismo Fernández Buey, habría una continuidad entre la idea de reciprocidad (algo muy distinto del principio de reciprocidad como condición de reconocimiento del derecho al voto para los inmigrantes, siguiendo al pie de la letra el mandato constitucional del artículo 13) y la del derecho a la participación política, es decir, a la condición (o a la equi- 
paración con esa condición) de ciudadanía. Aquélla, la reciprocidad, supone una suerte de contrato moral previo, prepolítico, que implica el derecho de crítica en los dos sentidos (del inmigrante y del miembro de la sociedad de recepción) y la aceptación de esa regla de juego de igualdad en el trato. Por su parte, la participación política aparece como una necesidad básica de todos aquéllos que forman parte establemente de una comunidad política, sea cual sea el grado de institucionalización o reconocimiento jurídico-político (estatus de ciudadanía plena o equiparacioón al mismo, por ejemplo). Es lo que Gil Calvo ha denominado un doble contrato social: un contrato de convivencia cívica que equipare en derechos civiles, sociales y políticos a los trabajadores a los que hemos llamado a nuestras sociedades, pero también un contrato de convivencia civil que permita la confianza mutua, en términos de «igual a igual», sin la que no hay cohesión y progreso social. Creo que, en buena medida, como he tratado de argumentar en otros trabajos, eso es lo que la Comisión Europea ha entendido como "ciudadanía cívica» en tanto que estatus a ofrecer a los inmigrantes residentes de larga duración en el territorio de la UE.

Es evidente que hablar de los nuevos sujetos de la integración política exige ampliar la participación en el espacio público plural y la propia extensión del mismo. El punto de referencia es la definición de ciudadanía y, desde ella, los cauces e instituciones de participación y presencia pública de los inmigrantes, con especial atención al reconocimiento y a la garantía de los derechos políticos, que no son sólo el derecho al sufragio, sino también los de participación. El modelo de gestión democrática de una sociedad plural como las nuestras, en la que los inmigrantes son un factor relevante y además representan aquello que genuinamente constituye el contrato democrático, pues nos han elegido como su sociedad de destino, no puede resistir por más tiempo esta exclusión institucionalizada, en la que una parte de quienes están y contribuyen a la sociedad y a las cargas del estado se ven privados de aquello que exige la democracia: el derecho, los derechos a intervenir y decidir sobre los criterios de gestión de la vida pública. Ésos son los derechos políticos, que van más allá del derecho al voto. Y esos derechos - la ciudadanía- deben poder estar al alcance de quienes quieren formar parte de nuestra sociedad.

Se nos abren así dos grandes debates. Uno, la necesaria revisión de la concepción de la ciudadanía, cuya versión clásica, la propia de los estados nación de los siglos XIX y XX, se encuentra cuestionada por la globalización, la creciente heterogeneidad que caracteriza a nuestras sociedades y una lectura consecuente con la hegemonía proclamada de los derechos humanos. El segundo debate hace referencia a la relación a establecer entre participación e integración de los inmigrantes. Uno y otro, en el caso europeo, remiten a la posibilidad de configurar una ciudadanía europea, de carácter plural e inclusivo. Desde una concepción de este tipo, la participación es un elemento clave y básico del proceso de integración de los inmigrantes, que, en ese sentido, podemos definir como el desarrollo de la participación de los nuevos ciudadanos en las diferentes esferas de la vida social, en igualdad de derechos y obligaciones y sin que se les imponga el precio de la renuncia a su cultura de origen. 


\section{Sobre la ampliación de la noción de ciudadanía}

Desde hace tiempo, he sostenido - en coincidencia con otros muchos - la legitimidad de un proceso de acceso a la ciudadanía, o al menos a la integración cívica, que es política, que comience en el ámbito municipal. Como otros (Bauböck, Rubio, Soysal), he formulado esa propuesta a partir de la vinculación entre ciudadanía y residencia, que debe entenderse en términos de inclusión, pluralidad y gradualidad. Lo que se trata de reivindicar es, en todo caso, un derecho, no una obligación, pero menos aún un privilegio ni una coartada que sirva para proletarizar y domesticar a esos sujetos. Un proyecto, insisto, que arranca de la noción de los vecinos, de los residentes, como ciudadanos de esas primeras comunidades políticas que son las ciudades, con todos los derechos y deberes de los demás vecinos, de los habitantes de las ciudades que llegaron antes que ellos y que, al ser nacionales del estado, son naturalmente ciudadanos. Ese proyecto contribuye a revisar las condiciones de reconocimiento y garantía de los derechos políticos en lo que se se refiere a esos new comers que son los inmigrantes. Pensar de nuevo en los criterios para definir la pertenencia, el título de soberanía y de derechos. Y eso significa volver a examinar nuestras respuestas acerca de los derechos de participación política. Una parte importante de esa respuesta, a mi juicio, pasa por incentivar los instrumentos de asociacionismo de y por los inmigrantes. En otros lugares me he ocupado de esa cuestión.

Se trata, por lo demás, de un argumento que cuenta con un considerable respaldo social y ciudadano, a través de movimientos e iniciativas de amplio espectro que coinciden en anclar el acceso a la ciudadanía en la residencia estable (desde tres años), en el ámbito local, es decir, ciudadanía como vecindad, con derechos políticos plenos en el ámbito municipal (lo que es algo más que el derecho a sufragio activo y pasivo, el derecho a voto), desde una concepción de la ciudadanía como ciudadanía multilateral (doble, y aún más, cuádruple ciudadanía) y, además, ciudadanía gradual: desde la vecindad al ámbito autonómico, primero, y al estatal y europeo, después.

Una concepción, además, que tiene apoyo en las recomendaciones de Tampere y en algunos pocos instrumentos - casi todos no normativosde política de inmigración de la Comisión, el Parlamento Europeo y el Comité Económico y Social. Un proyecto que trata de construir el proceso de lo que la propia Comisión ha definido como integración cívica y ciudadanía cívica. Esa ciudadanía cívica debe comenzar por el reconocimiento de que el residente (aunque sea sólo residente temporal y no definitivo o permanente), en la medida en que paga impuestos y contribuye, con su trabajo y con sus impuestos, con su presencia como vecino y no sólo como trabajador, a la construcción de la comunidad política, comenzando por la primera, la ciudad, tiene no sólo derechos civiles e incluso sociales, sino también políticos: derecho a participar al menos en ese nivel. El primer escalón de la ciudadanía cívica sería, de nuevo, el primer escalón de la idea europea, las ciudades, la comunidad política municipal. 
A mi juicio, la vía más adecuada para alcanzar ese objetivo es combinar ese principio de integración política con los de ciudadanía multilateral y ciudadanía local. A esos efectos, puede ser útil recuperar la noción de políticas de presencia, de participación en el espacio público, enunciada por Phillips, en relación con los grupos "desposeídos de poder", como propone Sassen y, en particular, los inmigrantes, las mujeres. En cierto que Sassen incluye en esa política de presencia dos objetivos diferentes, el de dar poder a los que están privados del acceso al poder y a la riqueza, y el de explicar la paradoja de la capacidad política creciente de grupos a los que se niega la titularidad de la ciudadanía. Por eso recurre a la noción de presencia y a la de ciudadanía de facto para tratar de superar la nacionalización de la ciudadanía y su contaminación de género. Más allá del interés de la propuesta específica de Sassen a propósito de la cuestión de género, me interesa señalar que sus sugerencias apuntan en la misma dirección que proponía. Se trata de abrir esas dos jaulas de hierro que aprisionan la ciudadanía, la del vínculo entre nacionalidad, trabajo formal y ciudadanía, y la de ciudadanía, espacio público y género. Se trata de crear nuevas formas de ciudadanía, plurales, multilaterales, y de carácter gradual, que conectan con la ciudadanía como «derecho a la ciudad», «derecho a la movilidad», "derecho a la presencia», sobre todo de quienes han sido arrinconados a los territorios donde oficialmente (al menos para quienes siguen sin entender a Foucault) no reside, no juega el poder, teniendo en cuenta que, desde esos espacios, esos actores - las mujeres, los inmigrantes, sobre todo los sin papeles - están tejiendo una nueva política.

En cuanto a los principios de ciudadanía múltiple o multilateral y local, como concreción de la democracia inclusiva y plural, lo que propongo es aprovechar las tesis defendidas por Bauböck o Rubio (y acogidas por Castles) a propósito de la ciudadanía transnacional, para definir la idea de ciudadanía o integración cívica antes enunciada. Se trata de una ciudadanía entendida no sólo en su dimensión técnico-formal, sino también social, capaz de garantizar plenos derechos civiles, sociales y políticos a todos los que residen establemente en un determinado territorio. La clave radica en evitar el anclaje de la ciudadanía en la nacionalidad (tanto por nacimiento como por naturalización), una identidad que pone de relieve la incapacidad de la propuesta liberal para superar las raíces etnoculturales del pretendido modelo republicano de ciudadanía. La ciudadanía debe regresar a su raíz y asentarse en la condición de residencia, por eso la importancia de la vecindad, de la ciudadanía local, que, por otra parte, es la que nos permite entender más fácilmente cómo los inmigrantes comparten con nosotros - los ciudadanos de la ciudad, los vecinos- las tareas, las necesidades, los deberes y, por tanto también, los derechos propios de ésta.

La dificultad, como apunté más arriba, estriba en cómo hacer asequible esa condición de residente estable equiparada a la de ciudadano, y hemos de discutir si debe tratarse de una condición que se adquiere simplemente tras un período consolidado de residencia (y, en ese caso, la duración del mismo: tres, cinco o más años) o si hace falta, además, superar un test de adaptación o inte- 
gración y de lealtad constitucional, tal y como, a la imagen de lo dispuesto en los EEUU, se ha establecido en recientes reformas en algunos de los países de la UE (pruebas de lengua, de conocimiento de la Constitución, de las costumbres y tradiciones del país).

Por mi parte, entiendo que debe tratarse de un efecto casi automático derivado de la estabilidad de residencia y la aceptación de la legalidad, a lo que cabe añadir el conocimiento de la lengua. Pese al carácter razonable de algunos de los requisitos enunciados, no puede ignorarse que todo lo que excede un principio simple, la libre aceptación del ordenamiento jurídico-constitucional, bordea peligrosamente un modelo de asimilación cultural como condición de la integración política. La receta es muy simple: a los inmigrantes, como a los ciudadanos, lo que se les debe exigir es el respeto o, mejor, el cumplimiento de la legalidad juridico-constitucional. Pero eso no significa que ellos deban ofrecer un plus que no se exige a los ciudadanos.

No es sólo un debate teórico. No faltan iniciativas concretas en ese sentido. Doce estados miembros - Austria, Dinamarca, Irlanda, Finlandia, Holanda, Suecia, la Republica Checa, Estonia, Hungría, Lituania, Eslovaquia y Eslovenia - ya conceden derecho a voto a nivel municipal a inmigrantes extracomunitarios sin distinción de nacionalidad, a los que habría que añadir la más reciente decisión en ese sentido, hace un año y medio, la del Parlamento de Bélgica. En aras de la brevedad y para concluir (dejando de lado algunas propuestas institucionales de interés, en particular las contenidas en los planes de inmigración de País Vasco, Andalucía y, más reciente y específicamente, Cataluña y el actual borrador de Plan de Ciudadanía e Integración que está discutiendo el Foro para la Integración Social de los Inmigrantes), me limitaré a mencionar algunas de las más recientes iniciativas que insisten en la participación política como elemento de la integración.

Mencionaré dos ejemplos a escala europea: las sugerencias incluidas en el capítulo segundo del Handbook on integration for policy makers and practitioners, elaborado por el MPG, coordinado por J. Niessen y Y. Schibel, y publicado por la Comisión Europea en noviembre de 2004, y el estudio comparado europeo en torno a cinco indicadores (mercado laboral, obtención de residencia, de nacionalidad, condiciones de reagrupamiento familiar y medidas antidiscriminación) contenido en el European Civic Citizenship and Inclusion Index, editado por el mismo MPG, British Council, en 2005. Por lo que se refiere al ámbito español, citaré, en primer lugar, la segunda parte del documento de buenas prácticas promovido en 2004 por el Real Instituto Elcano en colaboración con la FOG (Inmigración. Prioridades para una nueva politica española) relativa a integración. Además, el documento Dret de vot de les persones inmigrades, del Sindicato CCOO, presentado el 16 de diciembre de 2005 (http://www.conc.es/immigracio), y la iniciativa "Consenso social sobre las migraciones», promovida a lo largo, asimismo, de 2005, entre otras ONG, por Cáritas. Todas ellas incluyen un apartado específico sobre ciudadanía y participación política. En la propuesta de CCOO de Cataluña se reitera que la participación efectiva (y no sólo la participación social, también específicamente 
la política) es eje vertebrador de la cohesión social y del sentimineto de pertenencia, factor, por tanto, de integración y clave de una política proactiva de inmigración. Me parece interesante, asimismo, que, más allá de la medida concreta reivindicada (derecho al sufragio activo y pasivo en el ámbito municipal para los inmigrantes residentes legales que cuenten al menos con cinco años de residencia permanente en España), se destaque que la participación política va más allá del derecho al sufragio activo y pasivo (reivindicado de forma gradual, comenzando por el ámbito municipal), pues exige el derecho a intervenir para ser oído en el proceso de elaboración de las tomas de decisiones que les afecten, y requiere desde luego medidas de promoción y desarrollo del tejido asociativo (en particular, en el ámbito municipal: asociaciones de vecinos, culturales y escolares), como también garantías particularmente prioritarias por lo que se refiere a los derechos de sindicación, de asociación, de reunión y manifestación. Finalmente, recordaré que la última de esas propuestas exige «un consenso social para redefinir el concepto de ciudadanía, ya que el actual excluye a amplios colectivos [...] Si queremos construir una sociedad con futuro, hay que construir ciudadanía en igualdad de condiciones. Necesitamos un proyecto de convivencia compartido, un pacto de derechos y deberes concebido para todos y asumido por todos». En ese pacto de derechos y deberes, se pone un énfasis especial en el acceso a la vivienda, a la sanidad, a un puesto de trabajo formal, a una educación pública y plural y en la plena participación en la vida política y civil. Por eso, el proyecto del consenso social para la ciudadanía es definido como «un proceso de consenso social sobre las migraciones, que permita sustituir el concepto de extranjería por el de migración, que tenga en cuenta las dinámicas migratorias desde una perspectiva amplia, que conduzca a la implantación de políticas y que genere un modelo de integración propio y estable».

\section{La participación política de los inmigrantes, clave del proceso de integración}

Si no hay participación política — al menos en el sentido en el que lo propone la noción de ciudadanía cívica-, no hay sentimiento ni conciencia de identidad, de pertenencia. Si no se dan las condiciones para un protagonismo o una competencia efectivos de los inmigrantes en la elaboración y en la toma de decisión de lo que llamamos políticas públicas, difícilmente cabe hablar de integración social. Aún más, si no se logra crear el vínculo social y político, crear la conciencia de que no sólo se pertenece, sino que también se es reconocido como sujeto en el espacio público plural en condiciones de igualdad, no habrá integración. Menos todavía si pretendemos hablar en términos proactivos, desde una perspectiva de la integración como proceso que implica a todos los agentes presentes y protagonistas de las redes de relaciones sociales, es decir, no una integración unidireccional, sino mutua y global. Es hora, pues, de que analicemos las condiciones, los instrumentos, los programas que pueden hacer posible principios y prácticas de participación de los inmigrantes como sujetos, 
como parte activa, como protagonistas de este proceso de transformación social que debe venir guiado por la primacía de los derechos humanos, de la igualdad en esos derechos. Es en ese sentido que hablamos, claro, de condiciones, instrumentos y programas de la participación política. Cabría destacar tres aspectos: en primer lugar, el protagonismo de los propios inmigrantes y de los agentes públicos en la participación, lo que remite a un fenómeno de una importancia trascendental, el movimiento asociativo, el de los propios inmigrantes y también y en relación con ellos, el de las asociaciones (ONG) de solidaridad, ayuda y/o apoyo a los inmigrantes. En segundo lugar, lo que podríamos llamar la territorialización de la participación politica de los inmigrantes, la dimensión local —-municipal y autonómica - como contexto de partida del proceso gradual de participación política. En tercer lugar, es particularmente importante insistir en que, para que hablemos de participación política en serio, es necesario que se reconozca capacidad de decisión en la elaboración y el diseño de políticas públicas locales, municipales, autonómicas y estatales: el modelo de instituciones consultivas o asesoras (foros, consejos consultivos) que puede servir en una primera etapa, parece hoy ya abiertamente insuficiente.

La participación es siempre un proceso, y además implica como mínimo negociación y las más de las veces conflictos que pueden llegar a sustanciarse en sede jurisdiccional o, al menos, administrativa. En el caso de la participación política de los inmigrantes, no será de otro modo. Y hay que añadir que, pese a la vertiginosa rapidez con la que se está produciendo la transformación de España en un país de inmigración, los cambios que exige el reconocimiento de la participación política de los inmigrantes remiten a un proceso que implicará a varias generaciones.

Por lo demás, no es posible ignorar que las condiciones legales del proceso de participación no bastan de suyo. Las condiciones materiales de vida y de trabajo son decisivas a la hora de hacer posible proyectos de arraigo que permitan el desarrollo de mecanismos y cauces de participación en el espacio público y, sobre todo, que venzan la barrera del confinamiento, de la reducción al ámbito privado (a lo sumo, el laboral, en el que hay un primer paso de participación y presencia a través de la sindicación), en el que se encuentran buena parte de los inmigrantes residentes.

Aunque es verdad que la intervención en el espacio público empieza en la dimensión vecinal y laboral: los barrios (las propias viviendas, las asociaciones de vecinos), las escuelas (las asociaciones de padres y madres, las AMPA, porque la escuela es una institución que debe preparar y educar en la participación a todos los elementos de la comunidad escolar, a los estudiantes y a las familias) y los centros de trabajo (la afiliación y la participación sindical) son los tres primeros escalones de contribución, porque no hay que pensar sólo en redes formales e institucionales de participación. No se trata de incentivar sólo una vía propia de intervención. La creación (y el apoyo) de asociaciones propias de inmigrantes no puede ser el único objetivo. Se trata de remover las barreras que dificultan que éstos se integren en las diferentes manifestaciones 
del tejido asociativo y participativo, hasta incluir a las dos instituciones básicas: sindicatos y partidos. En ese sentido, resulta de la mayor importancia el análisis de la presencia, organización y participación de los inmigrantes en las organizaciones y entidades - y redes - religiosas, como también en las organizaciones deportivas y, en términos más generales, en las asociaciones juveniles.

Quizá la cuestión más llamativa radica en el hecho de que los vecinos residentes de origen extracomunitario no sean ciudadanos de su municipio. Viven en él, cumplen sus leyes y pagan sus impuestos, pero no forman parte del grupo de iguales que definen el gobierno de la ciudad con su voto. Esto plantea, entre otras cuestiones y de forma muy aguda, el debate sobre el derecho al sufragio, activo y pasivo, en el orden municipal, como palanca para mover la condición de los derechos políticos y de participación de los inmigrantes vecinos pero no ciudadanos. Ante la negación del derecho de sufragio local, el principio de participación proclamado en los diferentes planes municipales u otros documentos se ha concretado como participación social y cívica. Del conjunto de programas, líneas de trabajo y actuaciones que, bajo esa rúbrica, desarrollan los ayuntamientos, cabe destacar dos tendencias comunes: la creación de consejos municipales específicos, de tipo consultivo, y el fomento del asociacionismo inmigrante.

En los últimos años, han aumentado el número de consejos o foros municipales de inmigración, integración o convivencia, organismos consultivos, de base asociativa y que reúnen a representantes de organizaciones de inmigrantes, de acogida y del consistorio, técnicos y políticos. Este tipo de organismos se inscriben en la política de fomento de la participación ciudadana y la ciudadanía local, que, en la última década, ha adquirido una relevancia creciente en España. De hecho, desde hace años, ya funcionan consejos de estas características para otros grupos de vecinos (jóvenes, mujeres, tercera edad). Los consejos o foros de inmigración, que se rigen por la Ley de Bases de Régimen Local, tienen atribuidas una diversidad de funciones. Por un lado, se constituyen como un instrumento institucionalizado de participación de un grupo de población determinado: los vecinos inmigrantes, que, mediante los representantes de sus asociaciones, pueden plantear sus necesidades y problemas. Por otro lado, y al mismo tiempo, se trata de organismos de asesoramiento para la Corporación y de debate y coordinación entre los diferentes actores que intervienen en el proceso de inserción urbana de los nuevos vecinos (Administración local, inmigrantes y sociedad civil). Con la excepción de algún ayuntamiento pionero, como el de Barcelona, la experiencia práctica de este tipo de organismos es bastante corta, y su incidencia real y práctica parece muy desigual entre unos municipios y otros. Se trata de organismos meramente consultivos, cuya vitalidad y dinamismo dependen, de forma decisiva, de la voluntad política de la corporación y de los medios y los recursos asignados. Con todo, la inmensa mayoría de los técnicos municipales tienden a realizar una valoración positiva de este tipo de consejos subrayando su carácter de instrumento de interlocución, dialogo y coordinación para el mejor desarrollo de las actuaciones municipales. 
Cuando se habla de participación local, el fomento del asociacionismo inmigrante ha constituido otra línea de actuación desde las corporaciones locales, aunque llama la atención la diferencia entre la importancia proclamada del tema y los recursos y las actuaciones más bien modestas que se han dedicado a ello. Este fomento del asociacionismo se ha concretado como apoyo al surgimiento, a la consolidación y a la actividad de organizaciones de inmigrantes mediante fórmulas como la cesión de locales, la subvención de algunas actividades y las formas concretas de reconocimiento (la inclusión en el consejo municipal, la participación en determinados programas, etc.). Por otro lado, tenemos una migración muy reciente, que, como ha sucedido en otros países, privilegia el asociacionismo en organizaciones propias. Así, en una parte de nuestras ciudades, han surgido en los últimos años un conjunto de asociaciones de inmigrantes modestas, de ámbito local y con escasos recursos, en su inmensa mayoría, pero que cumplen importantes funciones para sus miembros y personas de sus colectivos de referencia. Hoy por hoy, estas asociaciones constituyen una realidad muy modesta, con una potencialidad de participación social poco desarrollada y cuya concreción práctica va a depender del arraigo y de la consolidación de estas organizaciones, de su relación con el tejido asociativo local y las instituciones de la ciudad de recepción.

En todo caso, parece evidente que la plena participación de los vecinos inmigrantes requiere medidas en los diversos planos: político, social y cívico. Así, el reconocimiento del derecho de sufragio activo y pasivo en el ámbito local (a fortiori en el autonómico, el estatal y el europeo) continúa constituyendo una asignatura pendiente. La igualdad política en el ámbito local es un aspecto clave, una condición necesaria aunque no suficiente, para evitar una división vecinal, impulsar la participación y la implicación con su entorno de los nuevos vecinos, facilitar la negociación de los necesarios ajustes entre todos los grupos de vecinos y fomentar, así, una cohesión social más igualitaria y respetuosa con la diferencia. En definitiva, para responder a la demanda de una nueva ciudadanía, adecuada a las exigencias de la democracia multicultural. Fue Tocqueville quien nos advirtió que «si los hombres han de seguir siendo civilizados o llegar a serlo, el arte de asociarse unos con otros debe crecer y mejorarse en la misma proporción en que se incremente la igualdad de condiciones». Pero es importante entender que esa igualdad de condiciones de la que habla Tocqueville como elemento clave para una sociedad civilizada, decente, junto al fortalecimiento del tejido y la dinámica asociativos, no es sólo una igualdad material, sino una igualdad en las condiciones de acceso al espacio público.

Creo que esa es la lección que podemos obtener y que no siempre se ha retenido en algunos de los debates a los que se ha asistido en el último año a propósito del fracaso de modelos de gestión de la diversidad cultural ligada a la inmigración. Me refiero, en primer lugar, al Reino Unido, que descubre asombrado nuevos viveros de fundamentalismo islámico y aun de terrorismo y de rechazo de la sociedad multicultural británica protagonizados por jóvenes educados desde su primera infancia en el british way of life, incluso jóvenes que 
han triunfado profesionalmente como titulados superiores. $Y$ sobre todo, con ocasión de los disturbios de las banlieues en Francia, en otoño de 2005. La revuelta de la banlieue permitió escuchar una reivindicación política, genuina, porque se denunciaba un déficit estructural de respeto, reconocimiento y representación y participación. Reivindicación y denuncia repetida por parte de los habitantes de esos suburbios y de esas ciudades desfavorecidos, olvidados por los poderes públicos y por los agentes económicos. Esa demanda se concretó en los cahiers de doléances presentados en octubre de 2006, un año después, ante la Asamblea Nacional y el Senado francés, por iniciativa de asociaciones como ACle Feu, fundada por dos familiares de Bouna Traoré y Zied Bena, los dos jóvenes muertos en Clichy-sous-Bois que estuvieron en el origen de la revuelta. Porque el común denominador de las reivindicaciones (hábitat, educación, empleo, transportes, infraestructuras urbanas...) es lo que se dio en denominar el «deficit de las tres R» (respeto, reconocimiento y representación). No habrá integración política, aunque se trabaje en medidas jurídicas y en mejora de las condiciones materiales, si el objetivo no es esa igualdad como sujetos en el espacio público, en el trabajo, en el mercado, en los media, en la acción política.

\section{Referencias bibliográficas}

Aja, E. y ARango, J. (2006). Veinte años de inmigración en España: Perspectiva jurídica y sociológica (1985-2004). Barcelona: Fundación CIDOB.

ÀLVAREZ, Ignasi (2002). «La construcción del inintegrable cultural». En: LUCAS, Javier de y TORRES, Francisco (eds.). Inmigrantes: ¿cómo los tenemos?: Algunos desafios y (malas) respuestas. Madrid: Talasa, p. 168-195.

BALIBAR (1994). «Qué significa la ciudadanía europea». Revista Internacional de Filosofia Política, núm. 4.

BAUBÖCK, R. (1994). Transnational citizenship: Membership and rights in international migration. Aldershot: Edward Elgar.

- (2002). «Political community beyond the sovereign state: supranational federalism and transnational minorities». En: VERTOVEC, S. y COHEN, R. (eds.).

- (2004). "Cómo transforma la inmigración a la ciudadanía». En: AUBARELL y ZAPATA (eds.). Inmigración y procesos de cambio. Barcelona: Icaria.

BORJA, J. y BELIL, M. (2001). Informe sobre participación ciudadana. Barcelona: Congrés de Municipis de Catalunya.

CASTElls, M. (2003). La era de la información. El poder de la identidad. Madrid: Alianza.

- (2004). Europa en construcción: Integración, identidades, seguridad. Barcelona: Fundación CIDOB.

CASTLES, S. (2004). «Globalización e inmigración». En: Aubarell y ZaPATA (eds.). Inmigración y procesos de cambio. Barcelona: Icaria.

GARCía AÑón, J. (2004). «Inmigración y derechos de ciudadanía». En: Aubarell y ZAPATA (eds.). Inmigración y procesos de cambio. Barcelona: Icaria.

Gauchet, M. (2000). Quand les droits de l'homme deviennent une politique. París: Le Débat.

Genro, T. (2003). Crisis de la democracia. Barcelona: Ediciones del Serbal. 
GeOrge, S. (2002). «Globalifóbicos no, ciudadanos globales sí». Foreign Affairs, núm. 2. Habermas, J. (1999). La inclusión del otro. Madrid: Paidós. Traducción y estudio preliminar de J. C. Velasco.

HALPER, J. (2002). «Accommodation, cultural space, contact zones and the management of diversity in situations of conflict: an anthropological Perspective». Ponencia expuesta en el International Symposium on Inmigration Policies in Europe and the Mediterranean celebrado en Barcelona. Publicado en: Aubarell y ZAPATA (eds.). Inmigración y procesos de cambio. Barcelona: Icaria.

Hammar, T. (1990). Democracy and the nation-state: Aliens, denizens and citizens in a world of international migration. Aldershot: Avebury.

LuCAS, J. de (2003). Globalización e identidades: Claves politicas y jurídicas. Barcelona: Icaria.

- (2004). «Ciudadanía, extranjería y derechos». En: Betegón, J.; PÁramo, J. R. de; LAPORTA, F. J. y PRIETO, L. (coords.). Constitución y derechos fundamentales. Madrid: Centro de Estudios Políticos y Constitucionales, p. 379-407.

- (2005). "Fronteras e identidades: paradojas del proyecto europeo». En el monográfico dedicado al tratado constitucional por la edición española de Le Monde Diplomatique: «Europa: momentos decisivos».

- (2006). «La integración de los inmigrantes: la integración política, condición del modelo de integración». En: LUCAS, J. de y DÍEZ, L. La integración de los inmigrantes. Madrid: Centro de Estudios Políticos y Constitucionales, p. 11-43.

MarTinello, M. (ed.) (1997). Migration, citizenship and ethno-national identities in the European Union. Aldershot: Ashgate.

Martínez de Pisón, J. y Giró Miranda, J. (coords.) (2003). Inmigración y ciudadanía: perspectivas sociojurídicas. Universidad de La Rioja. Servicio de Publicaciones.

MeZZADrA, S. (2005). Derecho de fuga: Migraciones, ciudadania y globalización. Madrid: Traficantes de Sueños.

Miraut, L. (ed.) (2004). Justicia, migración y derecho. Madrid: Dykinson.

REX, John (1995). "La metrópolis multicultural: la experiencia británica». En: LAMO DE ESPINOSA, Emilio (ed.). Culturas, estados, ciudadanos. Una aproximación al multiculturalismo en Europa. Madrid: Alianza Editorial, p. 197-223.

- (1996). "National Identity in the Democratic Multi-Cultural State». Sociological Research Online I, núm. 2. <http://www.socresonline.org.uk/socresonline/ $1 / 2 / 1 . h t m l>$

SARTORI, Giavonni (2000). La sociedad multiétnica: Pluralismo, multiculturalismo y extranjeros. Madrid: Taurus.

SASSEN, S. (2001). ¿Perdiendo el control? La soberanía en la era de la globalización. Barcelona: Bellaterra.

- (2003). Contrageografías de la globalización: Género y ciudadanía en los circuitos transfronterizos. Madrid: Traficantes de Sueños.

SEN, A. (2000). Repenser l'inégalité. París: Seuil.

SENNETT, R. (2003). El respeto, sobre la dignidad del hombre en un mundo de desigualdad. Barcelona: Anagrama.

SOLANES, A. (2002). «El padrón municipal como mecanismo de inclusión de los extranjeros indocumentados». En: BAÑo, J. M. y Climent, J. (coords.). Nuevas perspectivas del régimen local: Estudios en homenaje al profesor José M. Boquera Oliver. Valencia: Tirant lo Blanch.

SOlANES, A. (2004). «Las deficiencias de la política española sobre inmigración» y «Breve comentario sobre la reforma de la Ley de Extranjería y las últimas modifi- 
caciones que afectan al régimen jurídico del extranjero en España». En: VVAA. Inmigración, sociedad y estado: Una cuestión abierta. Junta de Andalucía. Consejería de Asuntos Sociales. Dirección General de Bienestar Social.

SolÉ, Carlota y PARELla, Sònia (2003): «Identidad colectiva y ciudadanía supranacional» ("Collective identity and supra-national citizenship»). Papeles de Economía Española, núm. 98, Madrid.

Solé, Carlota; Alcalde, Rosa; Pont, Joseph; Lurbe, Katia y Parella, Sònia (2002). «El concepto de integración desde la sociología de las migraciones». Migraciones, núm. 12.

SOYSAL, Y. (1994). Limits of citizenship: Migrants and postnational membership in Europe. Chicago: University of Chicago Press.

VVAA (2003). "Ciudadanía e inmigración». Anales de la Cátedra Francisco Suárez, núm. 37.

Whitol de Wenden, Catherine (1999). Limmigration en Europe. París: La Documentation Française.

- (1997). La citoyenneté européenne. París: Presses de Sciences Po.

ZAPATA, R. (2004). Multiculturalidad e inmigración. Madrid: Síntesis. Colección Ciencias Políticas.

- (2005). ¿Existe una cultura de acomodación en España? Barcelona: Documentos CIDOB. 